\title{
Management of metastatic thyroid cancer in pregnancy: risk and uncertainty
}

\author{
Christopher W Rowe1,4, Kirsten Murray', Andrew Woods2, Sandeep Gupta3,5, \\ Roger Smith ${ }^{1,4}$ and Katie Wynne ${ }^{1,4}$ \\ 1Departments of Endocrinology and Diabetes and 2Maternity and Gynaecology, John Hunter Hospital, \\ Newcastle, New South Wales, Australia, ${ }^{3}$ Department of Nuclear Medicine \& PET, Hunter New England \\ Imaging, John Hunter and Calvary Mater Hospital, Newcastle, New South Wales, Australia, \\ ${ }^{4}$ Schools of Medicine and Public Health, and ${ }^{5}$ Health Sciences, University of Newcastle, Newcastle, \\ New South Wales, Australia
}

\author{
Correspondence \\ should be addressed \\ to C Rowe \\ Email \\ Christopher.Rowe@ \\ hnehealth.nsw.gov.au
}

\section{Summary}

Metastatic thyroid cancer is an uncommon condition to be present at the time of pregnancy, but presents a challenging paradigm of care. Clinicians must balance the competing interests of long-term maternal health, best achieved by iatrogenic hyperthyroidism, regular radioiodine therapy and avoidance of dietary iodine, against the priority to care for the developing foetus, with inevitable compromise. Additionally, epidemiological and cellular data support the role of oestrogen as a growth factor for benign and malignant thyrocytes, although communicating the magnitude of this risk to patients and caregivers, as well as the uncertain impact of any pregnancy on long-term prognosis, remains challenging. Evidence to support treatment decisions in this uncommon situation is presented in the context of a case of a pregnant teenager with known metastatic papillary thyroid cancer and recent radioiodine therapy.

\section{Learning points:}

- Pregnancy is associated with the growth of thyroid nodules due to stimulation from oestrogen receptors on thyrocytes and HCG cross-stimulation of the TSH receptor.

- Thyroid cancer diagnosed during pregnancy has not been shown to be associated with increased rates of persistent or recurrent disease in most studies.

- There is little evidence to guide the management of metastatic thyroid cancer in pregnancy, where both maternal and foetal wellbeing must be carefully balanced.

\section{Background}

Thyroid cancer is the most common endocrine malignancy, and the second most common invasive malignancy complicating pregnancy, occurring in 1 of 1000 pregnancies (1). Compared with other solid organ malignancies such as breast cancer, thyroid cancer presents unique management challenges due to its usually slow proliferation rate, the efficacy of surgery and radioiodine as curative first-line treatment and poor response to standard chemotherapy. The majority of thyroid cancer patients present with localised disease, and widespread metastases are relatively uncommon, especially in women of childbearing age. The published reviews and case series of thyroid cancer in pregnancy focus predominantly on the more common situation of establishing the diagnosis of a suspicious thyroid nodule, and whether definitive therapy can be safely deferred until post-partum $(1,2)$. Thus, there is a paucity of information to guide clinicians caring for patients with metastatic thyroid cancer during 
pregnancy. We present the management of a pregnant patient with known metastatic papillary thyroid cancer (PTC) and recent radioiodine therapy and review the literature regarding risks of disease progression in pregnancy, endocrine management considerations and potential conflicts between maternal and foetal wellbeing.

\section{Case presentation}

The patient, a 16-year-old female, presented six weeks pregnant, having self-ceasing prescribed levothyroxine $(1250 \mu \mathrm{g} /$ week) at conception. Serum biochemistry confirmed pregnancy and noted a thyroid-stimulating hormone (TSH) level of 171.6IU/L (local first trimester reference range $0.4-2.5)$, undetectable free $\mathrm{T} 4$ and thyroglobulin $636 \mu \mathrm{g} / \mathrm{L}$ (reference $<30$ ).

Six years prior to this presentation (at age 10), she was diagnosed with sporadic PTC. There was no history suggesting a familial tumour syndrome, and she had no other medical history. Total thyroidectomy revealed a unifocal $35 \mathrm{~mm}$ left lobe tumour with extrathyroidal extension and ipsilateral lymph node metastases in the lateral neck. Post-operative $\mathrm{I}^{123}$-whole body scan (WBS) (Fig. 1A) identified residual uptake in the thyroid bed and contralateral lower neck, and she was treated with $I^{131}$ (3.01 GBq). Stimulated thyroglobulin was $786 \mu \mathrm{g} / \mathrm{L}$, and fell to $39 \mu \mathrm{g} / \mathrm{L}$ on levothyroxine (TSH 1.2 IU/L).

Imaging 12 months later (Fig. 1B) revealed iodine-avid lung metastases, treated with four further doses of $\mathrm{I}^{131}$, with the most recent dosimetry-adjusted dose of $9.9 \mathrm{GBq}$ after TSH withdrawal (Fig. 1E) administered 7 months prior to this presentation with pregnancy (lifetime cumulative dose 25.2GBq). Suppressed thyroglobulin level prior to this dose was $19 \mu \mathrm{g} / \mathrm{L}$.

\section{Treatment}

Between 6 and 14 weeks of gestation, extensive discussion occurred between the patient, her partner, her father and a multidisciplinary medical team. Issues discussed included profound hypothyroidism at the time of conception, risk of progression of metastases due to pregnancy and risks to maternal health due to delayed further treatment, risk of premature delivery due to maternal ill health and the guarded long-term prognosis of the patient. The patient decided to continue the pregnancy, supported by her partner and her father.

In addition to standard obstetric care for a highrisk pregnancy, levothyroxine was titrated aiming for TSH suppression $(<0.1 \mathrm{IU} / \mathrm{L})$ and a free $\mathrm{T} 4$ level close to the upper limit of the reference range for pregnancy. Dose escalation of levothyroxine was required from $1100 \mu \mathrm{g} /$ week at presentation to $1500 \mu \mathrm{g} /$ week by delivery. Control of TSH, and relationship with thyroglobulin during and after pregnancy, are summarised in Fig. 2. Maternal iron deficiency was managed with oral iron supplementation; however, a subsequent rise in TSH at this time raised the possibility of malabsorption of levothyroxine due to co-administration. Variable TSH levels despite administration reminders suggested variable adherence.

Due to risk of progression of pulmonary metastases and the potential for underlying pulmonary fibrosis from previous radioiodine ablations, respiratory status
A

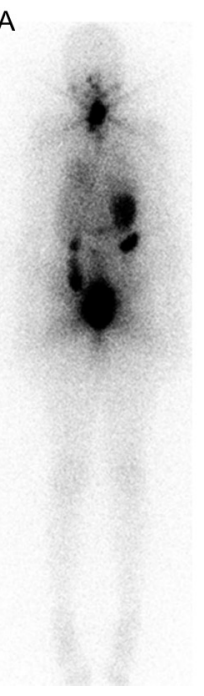

B

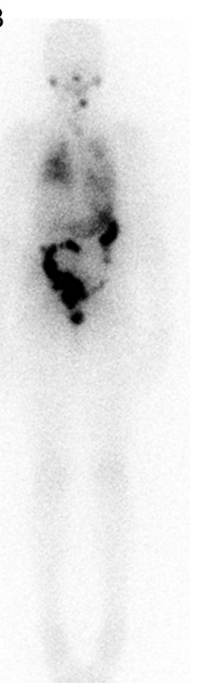

C

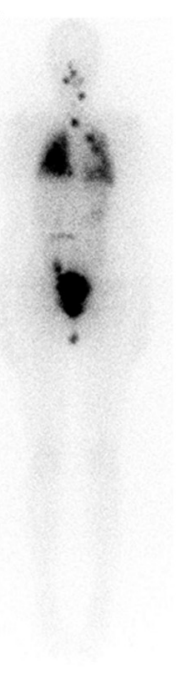

D

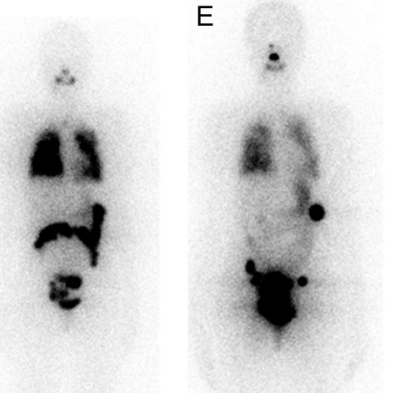

Figure 1

Serial post-I 131 therapy scans (anterior whole body views). (A) Age 10, 3.01 GBq; (B) age 11, 2.95 GBq; (C) age 13, 4.3 GBq; (D) age 14, 5.1 GBq; (E) age $15,9.9 \mathrm{GBq}, 7$ months before conception. Neck disease present at age 13 (C) was treated surgically. The final study (E) showed the presence of radioiodine avid bilateral pulmonary metastases $(<5 \mathrm{~mm}$ maximum diameter on computed tomography) and very small, low-grade lower neck disease. The focal uptake in the left upper abdomen is colonic and is likely physiological in nature. 


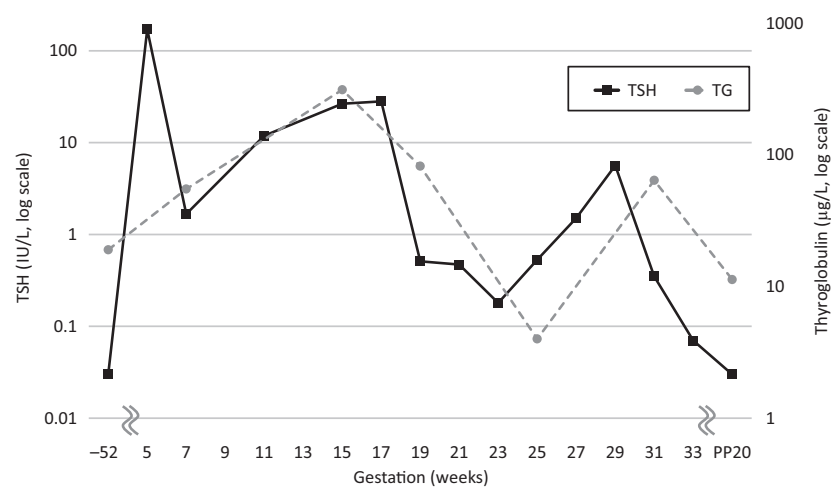

Figure 2

Relationship between serum TSH and thyroglobulin throughout gestation and post-partum. Levothyroxine replacement was adjusted at fortnightly intervals based on blood results. PP, post-partum.

was closely monitored by exercise tolerance, exertional pulse oximetry and formal lung function testing, without abnormality.

\section{Outcome and follow-up}

At 34 weeks of gestation, the patient presented in preterm labour, progressing rapidly to a normal birth of a healthy male child weighing $2380 \mathrm{~g}$. Breastfeeding was established on discharge, with an intention to continue for 3 months. The child's TSH was within normal limits. Maternal thyroglobulin 5 months post-partum was $11 \mu \mathrm{g} / \mathrm{L}$ with suppressed TSH. WBS after $3.8 \mathrm{GBq}$ of I131 administered 7 months post-partum continued to show radioiodine avid pulmonary metastases. Although there were some areas of increased focal uptake of radioiodine, the overall pulmonary uptake was not significantly different to the pre-pregnancy WBS. There were no new areas of abnormal radioiodine uptake. A concurrent CT chest showed maximum pulmonary nodule size of $3 \mathrm{~mm}$, unchanged from ante-partum.

\section{Discussion}

\section{Pregnancy and newly diagnosed thyroid cancer}

Thyroid cancer identified during pregnancy most commonly presents as a thyroid nodule, noted either coincidentally or with local symptoms. As the majority of cases are localised to the thyroid (1), the primary management decision is usually the timing of thyroidectomy - either during pregnancy or in the postpartum period - and whether the delay to any planned radioiodine therapy imposed by nursing or breastfeeding is acceptable. These issues are reviewed in recently published guidelines of the American Thyroid Association (2).

Several observations raise concern that pregnancy may reflect a proliferative environment for differentiated thyroid cancer (DTC). The known three-fold increased incidence of DTC in women of reproductive age suggests the involvement of reproductive hormones as thyroid growth factors, confirmed by cellular studies demonstrating proliferative effects of oestrogen on benign and malignant thyrocytes, mediated by oestrogen receptors (3). Studies of serial thyroid ultrasound in pregnant women demonstrate that pregnancy stimulates the formation and growth of thyroid nodules (4), although the absolute magnitude of nodule growth was small. Additionally, the glycoprotein hormone hCG can crossstimulate the TSH receptor due to structural homology and a common alpha subunit, which may mediate further stimulatory effects on thyrocytes.

Despite these concerns, most clinical outcome data show no difference in the rate of recurrence or long-term survival of women with well-differentiated thyroid cancer identified during pregnancy $(1,2,5)$. Two smaller studies noted DTC diagnosed during pregnancy was associated with higher rates of persistent disease and relapse, although possible confounders have been identified (2).

\section{Pregnancy and established DTC with known distant metastases}

DTC with distant metastases present during pregnancy is uncommon, occurring in $2-3 \%$ of pregnancies complicated by thyroid cancer $(1,5)$. To our knowledge, there are no specific guidelines for the prospective management of pregnancy in women with DTC with distant metastases identified prior to pregnancy or at conception. Recently, three large studies retrospectively examined the effect of pregnancy in survivors of thyroid cancer (2). Of 163 women studied, 9 had residual structural disease pre-conception (8 with cervical nodes, 1 with small volume lung metastases), highlighting the uncommon circumstances of our case. Thus, in the remainder of this article, we discuss clinical management principles for such patients, using parallels from the case vignette.

\section{Informed discussion of risk: balancing maternal and foetal wellbeing}

Active malignancy during a pregnancy may present a challenging management paradigm, with most decisions either prioritising maternal or foetal wellbeing, sometimes at the expense of the other (6). In pregnancy 
complicated by metastatic thyroid cancer, an important unquantifiable risk to maternal health is the potential for accelerated tumour growth due to proliferative effects of oestrogen, hCG and possibly progesterone on metastases, as discussed previously. Additionally, the duration of pregnancy and subsequent breastfeeding may delay planned radioiodine treatment and potentially result in disease progression. Radioiodine uptake is significantly increased in lactacting mammary tissue, and subsequent radiodioine treatment should be deferred for at least 3 months after cessation of lactation (2). Conversely, foetal health may be compromised by maternal thyroid status (both hypothyroidism and hyperthyroidism, discussed below), the potential for radiation exposure from residual radioiodine or the need for premature delivery in the case of maternal comorbidity.

This patient's large radioiodine dose 7 months prior to conception raises concern of residual radiation exposure to the foetus. Recent guidelines recommend deferring pregnancy for 6-12 months after radioiodine (2). Shorter time intervals may increase the risk of miscarriage; however, there is a lack of evidence demonstrating adverse pregnancy or foetal outcomes, including congenital abnormality.

\section{Levothyroxine replacement: goals, risks and pitfalls}

Physiologic requirements for thyroid hormone rise in early pregnancy due to doubling of thyroid binding globulin by oestrogen and expansion of plasma volume. Hypothyroid women must increase exogenous levothyroxine to compensate, usually by $30 \%$ at the time of conception (7), and pre-pregnancy counselling of such patients is mandatory. Monthly monitoring of thyroid function tests to allow early dose titration is recommended.

Maternal hypothyroidism during pregnancy may have deleterious effects on the foetus, particularly neurocognitive development. A study by Haddow (8) found that maternal hypothyroidism during pregnancy was associated with reduced IQ (mean 4 points) in offspring. However, this effect was most pronounced (mean IQ difference 7 points) in the subset of women whose hypothyroidism was untreated for the duration of pregnancy, and it is possible that prompt treatment may ameliorate this effect. Untreated hypothyroidism is also associated with increased risk of early pregnancy loss, and other obstetric complications including gestational hypertension, pre-eclampsia and preterm labour.

Although hyperthyroidism in pregnancy is associated with hypertension, low birth weight and preterm birth, subclinical hyperthyroidism with normal free thyroid hormones was not associated with an increased rate of adverse pregnancy outcomes in a large cohort study (9). In our patient, a suppressed TSH was targeted to minimise growth stimulus for cancer metastases; however, this may have contributed to premature labour. The strong correlation between TSH and level of thyroglobulin in this pregnancy (Fig. 2) supports the premise of TSH suppression to limit cancer growth in this patient.

Levothyroxine is poorly absorbed from the gut in the presence of iron and calcium supplements that are common components of pregnancy multivitamins. Our practice is to instruct patients to take levothyroxine on an empty stomach in the morning, and vitamins in the evening, to avoid inadvertent hypothyroidism due to malabsorption.

\section{Monitoring for disease progression: methods and endpoints}

Progression of metastatic DTC can be determined outside of pregnancy using structural imaging techniques such as computed tomography or functional imaging using radioiodine or ${ }^{18} \mathrm{~F}$-flurodeoxyglucose positron emission tomography. However, these modalities are not appropriate in pregnancy due to risk of ionising radiation to the foetus. Serial neck ultrasound is useful for monitoring for growth of any known structural disease in the neck and is safe in pregnancy.

Interpretation of serum thyroglobulin as a surrogate of tumour progression is complicated in pregnancy due to the stimulatory effect of physiologic fluctuations in TSH and thyroid hormone levels, and thus minor changes are unlikely to provide sufficient information to inform major clinical decisions (2). Widely variable TSH levels with corresponding changes in thyroglobulin were present in our patient, which confirmed tumoural TSH responsiveness but hindered meaningful assessment of tumour progression.

With these limitations, there was no objective evidence that this patient's pregnancy resulted in progression or worsening of her thyroid cancer.

\section{Prognosis of metastatic DTC}

Despite a variable prognosis, metastatic DTC has a favourable long-term survival compared to other cancers, particularly when diagnosed in childhood. A cohort study of 72 children with DTC (10), including 13 with lung metastases at diagnosis, found that 6 patients (including 2 patients presenting with pulmonary metastases) died over a median 13 years of follow-up, 12-33 years after initial 
treatment. The 10-year survival for all patients in this cohort was $98 \%$, falling to $90 \%$ at 20 years. Nonetheless, a standardised mortality ratio of 8.1 persists.

\section{Conclusions}

Metastatic DTC presents a challenging management paradigm in pregnancy. Ultimately, all decisions reflect an acceptance of the known and unknown risks balanced between mother and foetus, made through comprehensive engagement between clinicians in a multidisciplinary team, centred on the patient and her family. As similar cases are few, data to inform risk must be extrapolated from available evidence and clinician judgement.

\section{Declaration of interest}

The authors declare that there is no conflict of interest that could be perceived as prejudicing the impartiality of the research reported.

\section{Funding}

This work was supported by the Hunter-New-England Clinical Research Fellowship and the AVANT Doctors-in-Training Research Scholarship Program (to C R).

\section{Patient consent}

Written informed consent was obtained from the patient prior to publication

\section{Author contribution statement}

C R, K M, A W, S G and K W cared for the patient. C R drafted the manuscript with $\mathrm{K} \mathrm{W}$, with review and revision from $\mathrm{K} \mathrm{M}, \mathrm{A}$ W, S G and R S. All authors have reviewed the final manuscript.

\section{References}

1 Moosa M \& Mazzaferri EL 1997 Outcome of differentiated thyroid cancer diagnosed in pregnant women. Journal of Clinical Endocrinology and Metabolism 82 2862-2866. (doi:10.1210/jcem.82.9.4247)

2 Haugen BR, Alexander EK, Bible KC, Doherty GM, Mandel SJ, Nikiforov YE, Pacini F, Randolph GW, Sawka AM, Schlumberger M, et al. 20162015 American Thyroid Association Management guidelines for adult patients with thyroid nodules and differentiated thyroid cancer: the American Thyroid Association guidelines task force on thyroid nodules and differentiated thyroid cancer. Thyroid 26 1-133. (doi:10.1089/thy.2015.0020)

3 Derwahl M \& Nicula D 2014 Estrogen and its role in thyroid cancer. Endocrine-Related Cancer 21 T273-T283. (doi:10.1530/ERC-14-0053)

4 Kung AW, Chau MT, Lao TT, Tam SC \& Low LC 2002 The effect of pregnancy on thyroid nodule formation. Journal of Clinical Endocrinology and Metabolism 87 1010-1014. (doi:10.1210/jcem.87.3.8285)

5 Yasmeen S, Cress R, Romano PS, Xing G, Berger-Chen S, Danielsen B \& Smith LH 2005 Thyroid cancer in pregnancy. International Journal of Gynaecology and Obstetrics 91 15-20. (doi:10.1016/j. ijgo.2005.06.022)

6 Oduncu FS, Kimmig R, Hepp H \& Emmerich B 2003 Cancer in pregnancy: maternal-fetal conflict. Journal of Cancer Research and Clinical Oncology 129 133-146. (doi:10.1007/s00432-002-0406-6)

7 Alexander EK, Marqusee E, Lawrence J, Jarolim P, Fischer GA \& Larsen PR 2004 Timing and magnitude of increases in levothyroxine requirements during pregnancy in women with hypothyroidism. New England Journal of Medicine 351 241-249. (doi:10.1056/ NEJMoa040079)

8 Haddow JE, Palomaki GE, Allan WC, Williams JR, Knight GJ, Gagnon J, O'Heir CE, Mitchell ML, Hermos RJ, Waisbren SE, et al. 1999 Maternal thyroid deficiency during pregnancy and subsequent neuropsychological development of the child. New England Journal of Medicine 341 549-555. (doi:10.1056/ NEJM199908193410801)

9 Casey BM, Dashe JS, Wells CE, McIntire DD, Leveno KJ \& Cunningham FG 2006 Subclinical hyperthyroidism and pregnancy outcomes. Obstetrics and Gynaecology 107 337-341. (doi:10.1097/01. AOG.0000197991.64246.9a)

10 Schlumberger M, De Vathaire F, Travagli JP, Vassal G, Lemerle J, Parmentier C \& Tubiana M 1987 Differentiated thyroid carcinoma in childhood: long term follow-up of 72 patients. Journal of Clinical Endocrinology and Metabolism 65 1088-1094. (doi:10.1210/jcem-656-1088)

Received in final form 4 October 2016

Accepted 17 November 2016 